\title{
Metabolic Investigation of Patients with Staghorn Calculus: Is It Necessary?
}

\author{
Carmen R. P. Amaro, Jose Goldberg, Aparecido D. Agostinho, Patricia Damasio, Paulo R. \\ Kawano, Oscar E. H. Fugita, Joao L. Amaro
}

Lithotripsy Service (CRPA, PD) and Department of Urology (JG, ADA, PRK, OEHF, JLA), School of Medicine, UNESP, Botucatu, Brazil

\begin{abstract}
Objective: To evaluate the prevalence of metabolic disorders in patients with staghorn calculi treated at the Regional Center of Lithiasis Metabolic Studies in central region of São Paulo State, Brazil.

Materials and Methods: Between February 2000 and February 2008, 630 patients with urinary calculi were evaluated in the lithiasis outpatient clinic. Thirty-seven of them had staghorn calculi (35 women and 2 men). The inclusion criteria for the metabolic investigation included the absence of urological manipulation 30 days before the examination, negative urine culture and creatinine clearance $>60 \mathrm{~mL} / \mathrm{min}$. The protocol for metabolic investigation consisted of qualitative search for cystinuria. Two non-consecutive 24-hour urine samples collected to measure calcium, phosphorus, uric acid, sodium, potassium, magnesium, oxalate and citrate, and serum calcium levels, phosphorus, uric acid, sodium, potassium, magnesium, chloride, parathormone and urine $\mathrm{pH}$.

Results: Among patients with lithiasis, 5.9\% (37/630) had staghorn calculus and in 48.6\% (18/37) were diagnosed with urinary infection. The females were predominant for $94.5 \%$ of cases. The calculi were unilateral in 31 of cases and bilateral in six. Metabolic abnormalities were found in $68.2 \%$ of patients with hypercalciuria (64.2\%) and hypocitraturia (53.3\%) being the most common disorders.

Conclusions: The presence of metabolic disorders in nearly $70 \%$ of patients with staghorn calculus reinforces the necessity for evaluation of these patients. The diagnosis and treatment of identified metabolic abnormalities can contribute to the prevention of recurrent staghorn calculi.
\end{abstract}

Key words: lithiasis; calculus; metabolism; staghorn; evaluation

Int Braz J Urol. 2009; 35: 658-63

\section{INTRODUCTION}

Nephrolithiasis can develop as a result of metabolic abnormalities or anatomic malformations of the urinary tract and infection, as well as environmental and nutritional factors.

Staghorn calculus represents 10 to $20 \%$ of all nephrolithiasis cases. However, currently, in developed countries, this incidence has decreased con- siderably (4\%) due to early prevention and treatment of urinary infections (1). Its etiology is associated with urinary tract infection (UTI) caused by bacteria which produce ureases. It is characterized, in most cases, for having magnesium ammonium phosphate (struvite) or carbonate apatite in its composition (2), and for being more frequent in women. Besides urinary infection, other factors, which play an important role in the staghorn calculi formation, are anatomic 
alterations of the urinary tract such as stenosis of the pyeloureteral junction and neurogenic bladder, which cause lithiasis due to urinary stasis.

Oxalate or calcium phosphate calculi can initially occur in the kidney calices; and later take up the whole renal pelvis. This fact may be associated with no diagnosed and untreated metabolic disorders. In most cases, the optimal therapy is the complete eradication of calculus and urinary infection, correction of metabolic risk factors and any anatomic abnormalities (1). Our aim was to evaluate the likely metabolic alterations involved in staghorn calculi formation.

\section{MATERIALS AND METHODS}

Between February 2000 and February 2008, 630 lithiasic patients were prospectively evaluated in the outpatient clinic of metabolism of the Lithotripsy Service. Thirty-seven patients had staghorn calculus. A prospective protocol of urinary lithiasis investigation was used and the study was performed at least 30 days after any urological procedure. Complete staghorn calculi was considered when it occupied the renal pelvis and all calices systems, and incomplete when there was one in the pelvis and at least in one calyx. This study was approved by the Bioethics Commission of the School of Medicine - UNESP, Botucatu.

The inclusion criteria were normal renal function defined as creatinine clearance $>60 \mathrm{~mL} / \mathrm{min}$ and negative urine culture during the study period. Previous urinary infections, urinary tract malformation, family history of lithiasis and urological procedures to treat staghorn calculus were obtained through the registered clinical history.

The protocol of metabolic investigation consisted of qualitative search for cystinuria and two non-consecutive 24-hour urine samples collected to measure calcium, phosphorus, uric acid, sodium, potassium, magnesium, oxalate and citrate, in addition to serum levels of calcium, phosphorus, uric acid, sodium, potassium, magnesium, chloride, parathormone and fasting urine $\mathrm{pH}$. The protocol did not include calculus analysis.

Serum and urinary levels were performed at the Central Clinical Laboratory of the Clinic Hospital of the Medical School of Botucatu (UNESP).
The serum level was obtained from blood collected in dry tube with separator gel; after 30 minutes, it was centrifuged for 10 minutes at $300 \mathrm{rpm}$. The urinary $\mathrm{pH}$ was obtained through the $\mathrm{pHmeter}$, Micronal B371.

\section{RESULTS}

Among the patients with lithiasis, 37 (5.8\%) were diagnosed with staghorn calculus. The mean age was 44.2 years (range 25 to 68 years), there were 35 women and 2 men. Six patients presented bilateral calculi, and 31 unilateral cases.

Ten patients underwent a unilateral nephrectomy due to obstructive and infectious complications of staghorn calculus. In one case, stenosis of the pyeloureteral junction may have caused this calculus.

Fifteen patients, out of 37, presented complete staghorn calculus and underwent percutaneous nephrolithotomy or open surgery. Twenty-seven patients with incomplete calculi were submitted to extracorporeal shock wave lithotripsy.

Nearly half of them, 18 (48.6\%), presented clinical history of urinary tract infection, and only five were taking prophylactic antibiotics as a therapeutic measure. Bacteria found in previous medical records are shown in Table-1.

Twenty-two patients with staghorn calculus, who fulfilled the inclusion criteria, underwent metabolic investigation. Urinary $\mathrm{pH}$ mean was 6.25 (range $5.3-7.0$ ), and the mean of 24-hour urinary volume was $1,914 \mathrm{~mL}$ (range 830 - 3,500). Metabolic alterations (Table-2) were observed in $68.18 \%$ of patients

Table 1 - Incidence of different bacteria types identified in previous medical records.

\begin{tabular}{ll}
\hline Agent & N (\%) \\
\hline Proteus mirabilis & $5(27.7)$ \\
Escherichia coli & $4(22.2)$ \\
Klebsiella & $1 \quad(5.6)$ \\
Enteroccocus faecalis & $1 \quad(5.6)$ \\
Streptococcus & $1 \quad(5.6)$ \\
Non identified & $6(33.3)$ \\
\hline
\end{tabular}


Table 2 - Outcome of metabolic assessments in patients with staghorn calculus.

\begin{tabular}{lc}
\hline Metabolic Alterations & N (\%) \\
\hline Hypercalciuria & $9(64.2)$ \\
Hypocitraturia & $8(53.3)$ \\
Hyperuricosuria & $3(21.4)$ \\
Hypomagnesuria & $2(14.2)$ \\
Hyperoxaluria & $1(7.1)$ \\
Primary hyperparathyroidism & $1(7.1)$ \\
Renal tubular acidosis type 1 & $1(7.1)$ \\
\hline
\end{tabular}

$(15 / 22)$, and 9 of them presented concomitant history of urinary tract infection.

\section{COMMENTS}

Staghorn calculus is the most severe presentation of urinary tract lithiasis. Although UTI plays an important role in its formation, there are several other contributory factors, especially metabolic disorders. However, these disorders are not routinely investigated in these patients, and its frequency varies widely according to ethnical and environmental factors. Different studies of metabolic assessment in patients with urinary lithiasis have reported a high incidence of metabolic disorders as a causal factor $(3,4)$. Recently, Akagashi and others (5) evaluated 82 Japanese patients with staghorn calculus and found the following metabolic alterations: hypercalciuria in $37.8 \%$ and cistinuria in $2.4 \%$. Only $24.4 \%$ of patients had UTI, which is lower than the data from Western countries. As for gender, there was no difference, contradicting reports that show female predominance due to higher susceptibility of urinary tract infection.

Resnick and others (6) reported that, in Western countries, metabolic alterations correspond to $52.9 \%$ in men and $40.7 \%$ in women with staghorn calculus. These findings suggest that, in these countries, metabolic disorders usually coexist with urinary tract infection.

A Thai random study of 5,445 lithiasic patients observed frequent complete staghorn calculus in 86 of them $(1.6 \%)$. Positive urine culture was identified in $59.3 \%$ of patients, and hyperurecemia, the most common metabolic disorder, was found in $61.8 \%$ of patients with this calculus (7).

Staghorn calculus in children is usually related to congenial malformations of the urinary tract associated with infection and also metabolic diseases such as cystinuria, renal tubular acidosis and alterations like hypercalciuria and hyperuricosuria.

Ali and Rifat (8) studied 204 children with urinary lithiasis in Iraq, and identified the etiology in 189 (92.6\%), and the others were considered idiopathic. Twenty-nine patients (14.2\%) had staghorn calculus; 27 had history of urinary infection, and 25 had urinary tract malformation. Metabolic disorders were the most common cause, found in 106 children, and in $52(25.5 \%)$ they were associated with infection. Although metabolic disorders are the most frequent cause, its diagnosis can be difficult due to urinary tract infection.

As for patients with staghorn calculi and urinary tract infection, it is important to distinguish whether the infection was caused by ureases-producing bacteria that lead to calculus formation, especially the struvite, or just a complication due to metabolic disorders. Former reports have shown that 50\% of patients with calculus associated with infection presented metabolic disorders which are responsible for the initial calculus formation (5), showing the importance of metabolic investigation.

A study that evaluated biochemical risk factors concluded that the metabolic evaluation of patients with staghorn calculus seems to be important in the follow-up after surgical treatment (9).

There are limited publications related to the metabolic aspects of staghorn calculus formation and there are no Brazilian scientific publications focusing on the evaluation of this problem in our country. Our experience, from a regional reference center for calculi treatment, found $5.8 \%$ of patients with staghorn calculus from the total number sent for investigation of metabolic disorders. Because patients are not routinely sent for metabolic evaluation, this statistic may have been underestimated in our Service. There was female predominance due to characteristic factors related to their urinary tract. There were $27 \%(10 / 37)$ cases of nephectomies caused by lithiasis complications, and $18(48.6 \%)$ patients had a previous history 
of urinary tract infection. There was only one case of stenosis of the pyeloureteral junction.

Metabolic alterations were identified in 15 (68.2\%) of 22 patients evaluated, corroborating its importance as a contributing factor to staghorn calculus etiology. Thus, the presence of metabolic disorders in more than half of patients with this calculus highlights the importance of metabolic evaluation protocols. It is fundamental to correct metabolic disorders and control urinary infection in order to prevent recurrence of these calculi. Prevention is particularly difficult in these patients with recurrent infections. Therefore, they need careful follow-up with routine antibiotic therapy and urinary acidification control.

\section{CONCLUSION}

Metabolic analysis is important to be done mainly in the cases of staghorn calculus without urinary infection, however, this may not be the isolated etiology of these calculi. Thus, the clinical treatment of this disorder may be the only way to prevent its recurrence.

\section{CONFLICT OF INTEREST}

None declared.

\section{REFERENCES}

1. Rieu P: Infective lithiasis. Ann Urol (Paris). 2005; 39: 16-29.

2. Segura JW: Staghorn calculi. Urol Clin North Am. 1997; 24: 71-80.

3. Peres LA, Molina AS, Galles MH: Metabolic investigation of patients with urolithiasis in a specific region. Int Braz J Urol. 2003; 29: 217-20.

4. Amaro CR, Goldberg J, Amaro JL, Padovani CR: Metabolic assessment in patients with urinary lithiasis. Int Braz J Urol. 2005; 31: 29-33.

5. Akagashi K, Tanda H, Kato S, Ohnishi S, Nakajima H, Nanbu A, et al.: Characteristics of patients with staghorn calculi in our experience. Int J Urol. 2004; 11: $276-81$.

6. Resnick MI, Boyce WH: Bilateral staghorn calculi-patient evaluation and management. J Urol. 1980; 123: $338-41$.

7. Tanthanuch M: Staghorn calculi in southern Thailand. J Med Assoc Thai. 2006; 89: 2086-90.

8. Ali SH, Rifat UN: Etiological and clinical patterns of childhood urolithiasis in Iraq. Pediatr Nephrol. 2005; 20: 1453-7.

9. Wall I, Hellgren E, Larsson L, Tiselius HG: Biochemical risk factors in patients with renal staghorn stone disease. Urology. 1986; 28: 377-80.

\section{Correspondence address:}

Dr. João Luiz Amaro

Departamento de Urologia

Faculdade de Medicina de Botucatu

Botucatu, SP, 18618-970, Brazil

E-mail: jamaro@fmb.unesp.br 


\section{EDITORIAL COMMENT}

Etiologic investigation on urolithiasis is always of great interest because urologists often do not observe that the stone is a symptom and not a disease.

This Brazilian study underlines the high percentage of metabolic disorder (nearly $70 \%$ ) in patients with staghorn stones. This aspect is really interesting because usually we associate complex lithiasis with infection without classifying if the infection is established before or after stone formation.

The authors did not perform stone examination and we therefore assume that this aspect is integrated in the metabolic study of lithiasis, because for example the major presence of calcium oxalate or calcium phosphate (brushite) in a stone may orientate towards a metabolic alteration instead of an infectious cause like in a struvite or apatite stone. We must remember that this does not always have a simple interpretation because often stones are irregularly mixed.

Another important aspect that is the presence of metabolic alteration in patients who have undergone nephrectomies because if this is the case in most of them it suggests that a metabolic study should be mandatory.

In conclusion, we think that a metabolic study is necessary in all patients at risk for relapse like young, recidivated, multiple or bilateral lithiasis, familiarity, congenital or acquired solitary kidney and of course in patients with complex stones where often kidney function is reduced and we need to reduce possibility to develop a stone in contralateral kidney.

We hope that this field of study will not be abandoned because it may reduce patient suffering and health expenses due to relapse reduction.

\section{Dr. Mario Motta \& Dr. Alberto Saita Department Urology University of Catania, Italy E-mail:mmotta@unict.it}

\section{EDITORIAL COMMENT}

Most of the recent publications regarding staghorn calculi have focused on management issues. Thus, the study by Amaro et al. "Metabolic investigation of patients with staghorn calculi: Is it necessary?" is a welcome reminder that the cornerstone of therapy for recurrent nephrolithiasis is prevention. The authors screened a large population of adult stone patients, identifying 37 of 630 or $5.8 \%$ as having staghorn calculus. Half had a history of urinary tract infection. The overwhelming majority were women, a statistic that has been shown in previous epidemiologic studies of staghorn calculus. Of 22 patients who underwent metabolic evaluation, nearly $70 \%$ had a demonstrable metabolic abnormality including hypercalciuria, hypocitraturia, hypomagnesuria, and hyperuricosuria. This percentage is reasonably close to the percentage of patients identified with a metabolic abnormality in the non-staghorn population of stone patients (1). Why, then, would some individuals develop staghorn calculi and others not? It is possible that abnormal solute excretion is a risk factor for stone formation but that other factors determine the growth and presentation of the stones. Thus, some individuals may have a single stone, others have multiple bilateral stones, still others have enormous space-filling staghorn calculi. One of these factors may be the occurrence and bacte- 
riology of urinary tract infections. Another possibility is that the characteristics of the uroepithelium or urine contents of patients who develop urinary tract infections are similar to those with patients who develop stones. Uromodulin abnormalities, for example, have been implicated in the development of both stones and urinary tract infections (2).

It would have been of interest to know the composition of the staghorn calculi and to determine the correlation between staghorn stone composition and the urinary metabolic analysis. For example, was hypercalciuria seen in patients who had only calcium oxalate or calcium phosphate stones or was it also associated with the development of struvite stones? Despite the absence of these data, this study reminds us that stones develop as a result of abnormal supersaturation and that prevention depends in large part on limiting urine solute excretion and/or concentration. While we are looking for the ideal techniques for management of this very challenging condition, let us not forget the basics of prevention.

\section{REFERENCES}

1. Maloney ME, Springhart WP, Ekeruo WO, Young MD, Enemchukwu CU, Preminger GM: Ethnic background has minimal impact on the etiology of nephrolithiasis. J Urol. 2005; 173: 2001-4.

2. Serafini-Cessi F, Malagolini N, Cavallone D: TammHorsfall glycoprotein: biology and clinical relevance. Am J Kidney Dis. 2003; 42: 658-76.

Dr. Eleanor D. Lederer Director, Nephrology Training Program Kidney Disease Program, University of Louisville Louisville, Kentucky, USA E-mail: e.lederer@louisville.edu 